\section{Desempenho da vigilância de contatos de casos de hanseníase: uma análise espaço- temporal no Estado da Bahia, Região Nordeste do Brasil}

\section{Performance of leprosy case-contact surveillance: a space-time analysis in Bahia State, Northeast Brazil}

\section{Desempeño de la vigilancia de contactos en casos de lepra: un análisis espacio-temporal en el estado de Bahía, región nordeste de Brasil}

Eliana Amorim de Souza 1,2

Anderson Fuentes Ferreira 2

Maria Solange Araújo Paiva Pinto 2

Jorg Heukelbach 2,3

Héllen Xavier Oliveira 3

Jaqueline Caracas Barbosa 2,4

Alberto Novaes Ramos Jr. 2,5

doi: 10.1590/0102-311X00209518

\section{Resumo}

Objetivou-se analisar a tendência espaço-temporal da proporção de contatos examinados entre os registrados, segundo perfil demográfico de casos novos de hanseníase diagnosticados no Estado da Bahia, Brasil, na coorte 2003-2014. Trata-se de estudo ecológico de base populacional estadual, com análises temporal e espacial da proporção de contatos examinados entre os registrados segundo características do caso referência de hanseníase, com base no Sistema de Informação de Agravos de Notificação do Ministério da Saúde. A análise de tendência temporal foi baseada na regressão de Poisson por pontos de inflexão (Joinpoints). A análise espacial utilizou a autocorrelação espacial pelo Índice Local de Associação Espacial. Ao longo da série histórica, 52,9\% (55.116/104.142) dos contatos registrados foram examinados, com menor proporção entre pessoas < 60 anos, raça/etnia indígena e residentes em municípios de grande porte (em particular na capital). Verificou-se tendência de aumento da proporção de contatos examinados, com menor expressão quando o caso referência de hanseníase era do sexo masculino, de raça/cor preta, residente em zona rural e em cidades de pequeno porte, além da capital do estado. A distribuição espacial demonstrou que a maioria dos municípios apresentou desempenho com parâmetro precário, com aglomerados identificados nas regiões norte e extremo-sul. O Estado da Bahia apresenta desempenho precário das ações de vigilância de contatos, em particular diante de condições de maior vulnerabilidade social. Estratégias adicionais devem ser implementadas com vistas à superação de obstáculos operacionais para essa ação, considerada essencial para a interrupção da transmissão da hanseníase.

Hanseniase; Busca de Comunicante; Vigilância em Saúde Pública;

Prevenção \& Controle

\section{Correspondência}

E. A. Souza

Instituto Multidisciplinar em Saúde, Universidade

Federal da Bahia.

Rua Hormindo Barros 58, Quadra 17, Lote 58, Vitória da Conquista, BA 45029-094, Brasil.

eliana.amorim@ufba.br

1 Instituto Multidisciplinar em Saúde, Universidade Federal da Bahia, Vitória da Conquista, Brasil.

2 Programa de Pós-graduação em Saúde Pública, Universidade Federal do Ceará, Fortaleza, Brasil.

3 College of Public Health, Medical and Veterinary Sciences, James Cook University, Townsville, Australia.

4 Netherlands Hanseniasis Relief do Brasil, Fortaleza, Brasil.

5 Departamento de Saúde Comunitária, Universidade Federal

do Ceará, Fortaleza, Brasil. 


\section{Introdução}

A detecção precoce de casos de hanseníase e o tratamento oportuno e adequado com a poliquimioterapia (PQT) são as bases para o seu controle 1,2. A despeito de sua relevância, a doença persiste em posição de elevada endemicidade em países da Ásia, América do Sul e África ${ }^{3}$. Seu caráter focal de ocorrência no espaço revela, mesmo em países com baixa endemicidade, a possibilidade de existir aglomerados com alta carga de doença 4. Portanto, torna-se fundamental o desenvolvimento de novas estratégias que possibilitem a interrupção da transmissão da doença e a redução da sua detecção de forma sustentada e eficiente 1,5 .

O seu controle requer a busca ativa de casos suspeitos, conjuntamente com ações já estabelecidas como as de profilaxia pré (vacina BCG), além de ações em processo de validação, como a pós-exposição (quimioprofilaxia) para contatos de pessoas diagnosticadas como caso novo de hanseníase. Ademais, é estratégico o desenvolvimento e o aperfeiçoamento de metodologias diagnósticas para identificar aquelas pessoas sob maior risco de adoecimento 5 . Por outro lado, torna-se fundamental o planejamento de ações integradas e resolutivas para o cuidado a famílias e suas redes sociais acometidas pela hanseníase no território da atenção primária à saúde (APS) 2,6. Por tratar-se de evento negligenciado, reduzir contextos de desigualdades e iniquidades sociais também demanda a composição de estratégias para impedir adoecimento, estigma e incapacidade física associados 7,8 .

Durante a investigação epidemiológica de casos novos da doença é possível não encontrar relação com outro caso reconhecido na rede de convívio, embora possivelmente todos tenham sido expostos a uma fonte ativa, em algum momento de suas vidas 9,10. Por outro lado, muitos casos não identificados estão de fato relacionados a casos recém-diagnosticados e poderão permanecer sem diagnóstico, se não ocorrer adequada avaliação de seus contatos 11,12. Assim, entre as ações de controle, a adequada abordagem de contatos intra e extradomiciliares contribui sobremaneira com proporção significativa de casos novos, de forma especial, em contextos com endemicidade relativamente baixa ou moderada $11,12,13$.

Nesse sentido, a Estratégia Global da Organização Mundial da Saúde (OMS) para os períodos 2011-2015 e 2016-2020 recomendam o fortalecimento dessas ações para acelerar o controle da doença 1. Como condição crônica, infecciosa e negligenciada, a abordagem do contato constitui um momento significativo para a saúde pública, na medida em que tem o potencial de gerar aconselhamento e seguimento longitudinal sistemático de pessoas e famílias expostas ao risco de adoecimento 1,2,5.

Porém, é necessário considerar aspectos operacionais, logísticos, econômicos e éticos que estão envolvidos no processo de identificação e seguimento dos contatos, como revelação do diagnóstico do caso de hanseníase, capacitação dos profissionais da rede de atenção e análise de custo-efetividade das ações desenvolvidas. Dessa forma, o diagnóstico precoce da hanseníase é mantido como desafio para gestores e profissionais de saúde, em especial na APS 14,15.

Com o intuito de monitorar e avaliar as ações desenvolvidas por serviços de saúde para o controle, o Ministério da Saúde preconiza o uso de indicadores operacionais. Atualmente, o principal indicador utilizado para a avaliação da vigilância de contatos é, nos anos das coortes, a proporção de contatos examinados de casos novos de hanseníase diagnosticados entre os contatos registrados 2 . O desempenho do município é considerado bom quando esse indicador alcança a proporção $\geq 90 \%$. No Brasil, a média de contatos examinados entre 2012 e 2016 foi de $77 \%$, desempenho considerado regular $(\geq 75 \%$ a $89,9 \%$ ), já na Bahia a média foi de $67,1 \%$, dentro do parâmetro de desempenho precário $(<75 \%) 2,16$.

Considerando a relevância do exame de contatos para o controle da hanseníase, assim como as diferentes dimensões de vulnerabilidades envolvidas na manutenção da elevada carga e alta transcendência da hanseníase no Nordeste do Brasil, o presente artigo buscou analisar a tendência espaçotemporal da proporção de contatos examinados, com base no perfil demográfico dos casos novos de hanseníase diagnosticados no Estado da Bahia nos anos da coorte de 2003 a 2014. 


\section{Métodos}

\section{Local do estudo}

O Estado da Bahia está localizado no Nordeste do Brasil, região historicamente marcada por forte desigualdade social. Com uma população estimada em 14.812.617 habitantes, para o ano de 2018, tem $51,8 \%$ da população do sexo feminino, 59,3\% de cor parda, 27,8\% na faixa etária de 15 a 29 anos, 14,7\% analfabetos e 75,1\% residentes na zona urbana, sendo mais frequentes os municípios de pequeno porte 17. Destaque-se que a população indígena da Bahia totaliza 55 mil pessoas $(0,5 \%)$, residentes principalmente nas regiões sul e extremo-sul do estado. O contexto geral da Bahia é desafiador considerandose não apenas sua extensa dimensão geográfica $\left(564.831 \mathrm{~km}^{2}\right)$, como também contextos de pobreza em diferentes regiões e municípios. Embora ocupe o 8o lugar no ranking relativo ao Produto Interno Bruto (PIB) no Brasil, é o mais desigual dos estados da Região Nordeste, com índice Gini de 0,506 18. Do ponto de vista administrativo, os 417 municípios baianos compõem nove regiões de saúde (Figura 1).

\section{Figura 1}

Regiões de saúde do Estado da Bahia, Região Nordeste do Brasil.

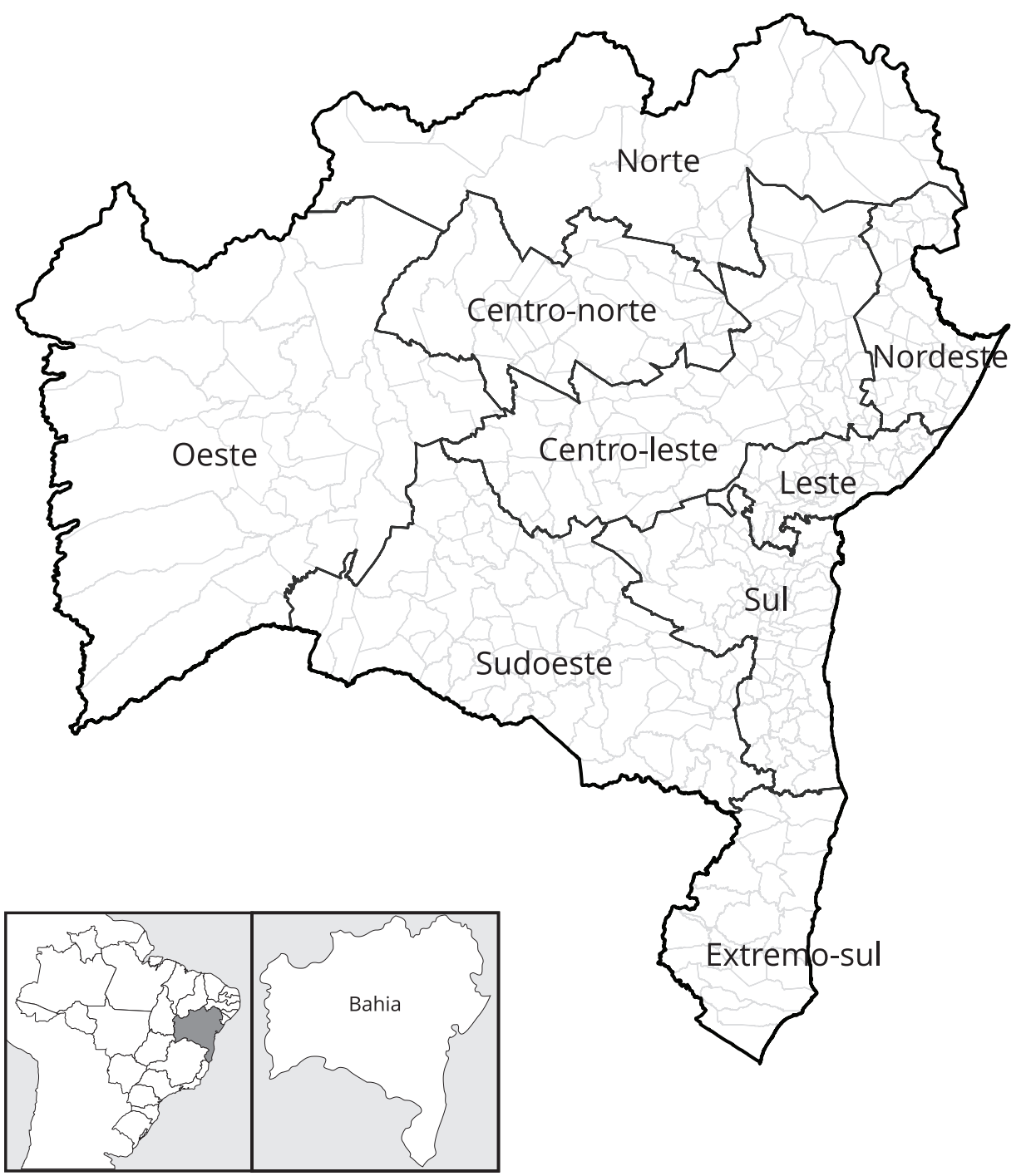




\section{Desenho de estudo e fonte de dados}

Trata-se de um estudo ecológico misto, com abordagens temporal e espacial, baseado na análise de dados secundários dos casos novos de hanseníase, valendo-se das bases de dados do Sistema de Informação de Agravos de Notificação (SINAN). O acesso ao banco de dados foi obtido junto à Diretoria de Informação em Saúde do Estado da Bahia (Secretaria de Estado da Saúde), com a inclusão de todos os casos notificados na coorte de 2003 a 2014, residentes no estado. Foram excluídos os casos que apresentaram como critérios de saída "erro diagnóstico". Definiu-se caso-referência (CR) como o caso de hanseníase notificado que serviu de base para a análise do desempenho do exame de contatos intradomiciliares. Também por normatização do Ministério da Saúde até 2016, contato domiciliar foi conceituado como toda e qualquer pessoa que resida ou tenha residido com o caso de hanseníase até cinco anos antes do seu diagnóstico 2.

\section{Análise descritiva}

Para a caracterização do CR de hanseníase, aos quais estão vinculados contatos registrados/examinados, foram utilizadas as seguintes variáveis: sexo (masculino e feminino), raça/cor (branca, parda, preta e indígena), faixa etária em anos $(<15,15$ a 60 e $\geq 60$ ), escolaridade (analfabeto, 1a a 4a série, 5a a 8a série, Ensino Médio, Ensino Superior), residência na capital do estado, zona de residência (urbana, rural e periurbana) e porte do município (pequeno porte: < 100 mil habitantes, médio porte: 100-500 mil habitantes e grande porte: $>500$ mil habitantes).

O cálculo da proporção de contatos examinados entre os contatos registrados nos anos das coortes seguiu a normativa do Ministério da Saúde 2. Dessa forma, considerou-se para os contatos intradomiciliares de casos novos de hanseníase com classificação operacional paucibacilar, aqueles com data de diagnóstico de 1 ano antes, e para os casos multibacilares, diagnóstico de até 2 anos antes. Assim, a série histórica compreendeu o período de 2003 a 2014.

\section{Tendência temporal}

A análise da tendência temporal da proporção de contatos examinados entre os registrados foi realizada segundo as variáveis sociodemográficas do CR de hanseníase. Utilizou-se a regressão de Poisson por pontos de inflexão - Joinpoint, por intermédio do Joinpoint Regression Program, versão 4.4.2 (http://surveillance.cancer.gov/Joinpoint/). Essa técnica estatística proporciona o ajuste de uma série de linhas, bem como de seus pontos de inflexão, em uma escala logarítmica com teste de tendências anuais. Para a obtenção do ajuste baseado na melhor linha de cada um dos segmentos analisados, utilizou-se o método de permutação de Monte Carlo como teste de significância. Baseando-se na definição dos segmentos, estimou-se e testou-se a Variação Percentual Anual (Annual Percentual Change APC) e a Variação Percentual Anual Média (Average Annual Percentual Change - AAPC), com seus respectivos intervalos de $95 \%$ de confiança (IC95\%).

O resultado ao longo de toda a série histórica indica tendência de crescimento, com valores de APC positivos, e de redução com valores de APC negativos. A ausência de tendência é estabelecida com o valor de APC igual a 0 (hipótese nula, $\mathrm{APC}=0$, taxas sem tendência definida).

\section{Análise espacial}

Para a análise espacial calculou-se a proporção média de contatos examinados entre os registrados por município, para o período total da coorte (2003-2014) e para dois subperíodos (2003-2008 e 2009 2014). A definição desses intervalos deveu-se às pequenas alterações na distribuição espacial quando usados intervalos de tempo menores. Os parâmetros empregados para a classificação do desempenho dos municípios por esse indicador foram baseados nos referenciais do Ministério da Saúde: precário - proporção de contatos examinados < 75\%; regular $\geq 75 \%$ e < 90\%; e bom > 90\% 2 .

Com o objetivo de identificar possíveis áreas de autocorrelação espacial, utilizou-se a análise do Índice Moran Local (Local Index of Spatial Association - LISA) 19. Calculou-se o indicador "invertido”, ou seja, a proporção de contatos não examinados entre os registrados. Dessa forma, gerou-se o 
diagrama de espalhamento no qual os municípios com alta proporção de contatos não examinados, circundados por municípios com condição similar, poderiam formar cluster (Q1: Alto-Alto) de desempenho ruim. Municípios com baixa proporção de contatos não examinados, tendo em seu entorno municípios com condição similar (Q2: Baixo-Baixo), indicariam aglomerados com melhor desempenho. Em um espectro intermediário estão municípios com alta ou baixa proporção de contatos não examinados circundados, respectivamente, por municípios com baixa ou alta proporção de contatos não examinados (Q3: Alto-Baixo e Q4: Baixo-Alto). Para a representação espacial, usou-se o Moran Maps considerando aqueles municípios com diferença estatisticamente significante.

Para a extração dos dados do SINAN utilizou-se o software Tabwin, versão 3.6 (http://www2. datasus.gov.br/DATASUS/index.php?area=060805) e para o cálculo da proporção de contatos avaliado na coorte, o software Stata, versão 11.2 (https://www.stata.com). Os softwares ArcGIS, versão 9.3 (http://www.esri.com/software/arcgis/index.html), e Terra View, versão 4.1 (http://www.dpi.inpe. br/terraview), foram utilizados para processamento, análise, apresentação dos dados cartográficos e cálculo dos indicadores de autocorrelação espacial, bem como para a construção de mapas temáticos.

\section{Aspectos éticos}

O estudo respeitou a Resolução no 466 do Conselho Nacional de Saúde, de 12 de outubro de 2012, e foi aprovado pelo Comitê de Ética em Pesquisa da Universidade Federal do Ceará, sob o parecer 544.962 de 28 de fevereiro de 2014.

\section{Resultados}

Para os 30.523 casos novos de hanseníase que compuseram a coorte de 2003 a 2014 no Estado da Bahia, foram registrados 104.142 contatos, entre os quais $55.116(52,9 \%)$ foram examinados. Ao longo da série histórica, houve ampliação da proporção de contatos examinados entre os registrados, de 44,6\% em 2003 para 68,8\% em 2014. Porém, essa ampliação ocorreu de forma distinta segundo características demográficas dos CR de hanseníase.

Para o sexo do CR, a proporção de contatos examinados entre os registrados foi próxima de 69,6\% para os homens e de $68 \%$ para as mulheres (Figura 2a). Maior proporção foi verificada quando o CR tinha $\geq 60$ anos (71,3\%) (Figura 2 b) e referiu raça/cor parda (71,8\%). Destaca-se a raça/cor indígena que variou de 23,5\% em 2005 para 68,7\% em 2014, apesar da expressiva variação na série histórica (Figura 2c).

Residir na capital do Estado da Bahia implicou menor proporção de contatos examinados, variando de 15,1\% em 2003 para 33,7\% em 2014 (Figura 3a). Nesse último ano, para contatos de CR que residiam fora da capital, 73,1\% foram examinados. Residir em região periurbana apresentou as menores proporções até 2011, com ampliação posterior, alcançando 91,5\% em 2014 (Figura 3b). Menores proporções na coorte foram verificadas quando o CR residia em cidades de grande porte, variando de 16\% em 2003 a 44,1\% em 2014. Nesse último ano, em municípios de médio porte, a proporção foi de $80,2 \%$ (Figura 3c).

A tendência temporal por Joinpoint foi de aumento na proporção de avaliação de contatos quando estes eram vinculados ao CR do sexo feminino (APC = 5,3; IC95\%: 3,4; 7,3). Entre contatos examinados vinculados ao CR do sexo masculino, houve tendência de redução significativa do indicador até 2005 (APC = -14,0; IC95\%: -24,3; -2,4), com aumento posterior até 2008 (APC = 14,2; IC95\%: 0,2; 30,1), ano a partir do qual verificou-se tendência de aumento significativo (APC = 4,0; IC95\%: 2,1; 5,9). Ao longo de toda a série histórica a AAP foi de 4,9 (IC95\%: 3,0; 6,8) (Tabela 1).

Não houve tendência definida para a idade na proporção de contatos examinados vinculados ao CR menor de 15 anos (APC = 3,8; IC95\%: -0,1; 7,8), diferentemente das demais faixas etárias que registraram aumento significativo, em particular quando o CR tinha entre 15 e 60 anos (APC = 5,4; IC95\%: 3,9; 6,9). Para a faixa etária > 60 anos, foram identificadas três tendências significativas, a primeira de redução no período de 2003-2005 (APC =-18,2; IC95\%: -31,1; -3,0) e a segunda e a terceira de incremento, respectivamente, 2005-2008 (APC = 7,1; IC95\%: 4,3; 9,9) e 2008-2014 (APC = 4,1; IC95\%: 1,6; 6,7) (Tabela 1). 


\section{Figura 2}

Proporção de contatos examinados entre os registrados, segundo variáveis demográficas dos casos-referência de hanseníase. Bahia, Brasil, 2003-2014.

2a) Sexo

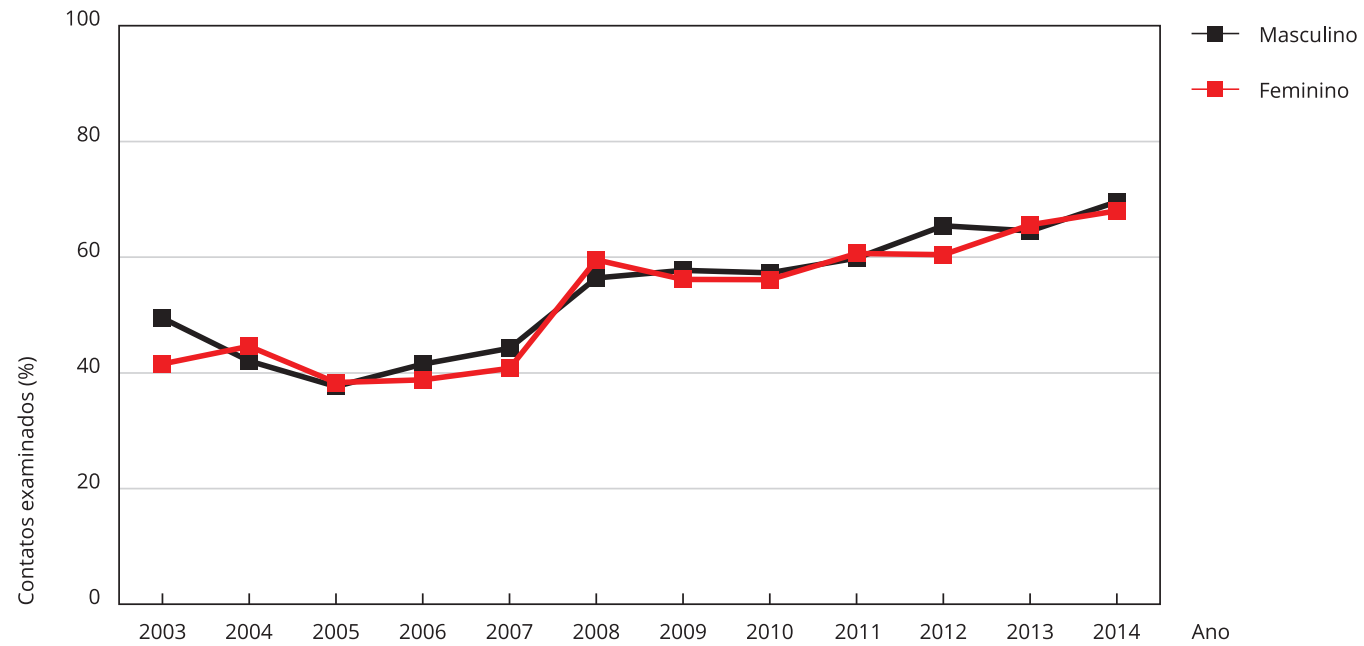

2b) Faixa etária

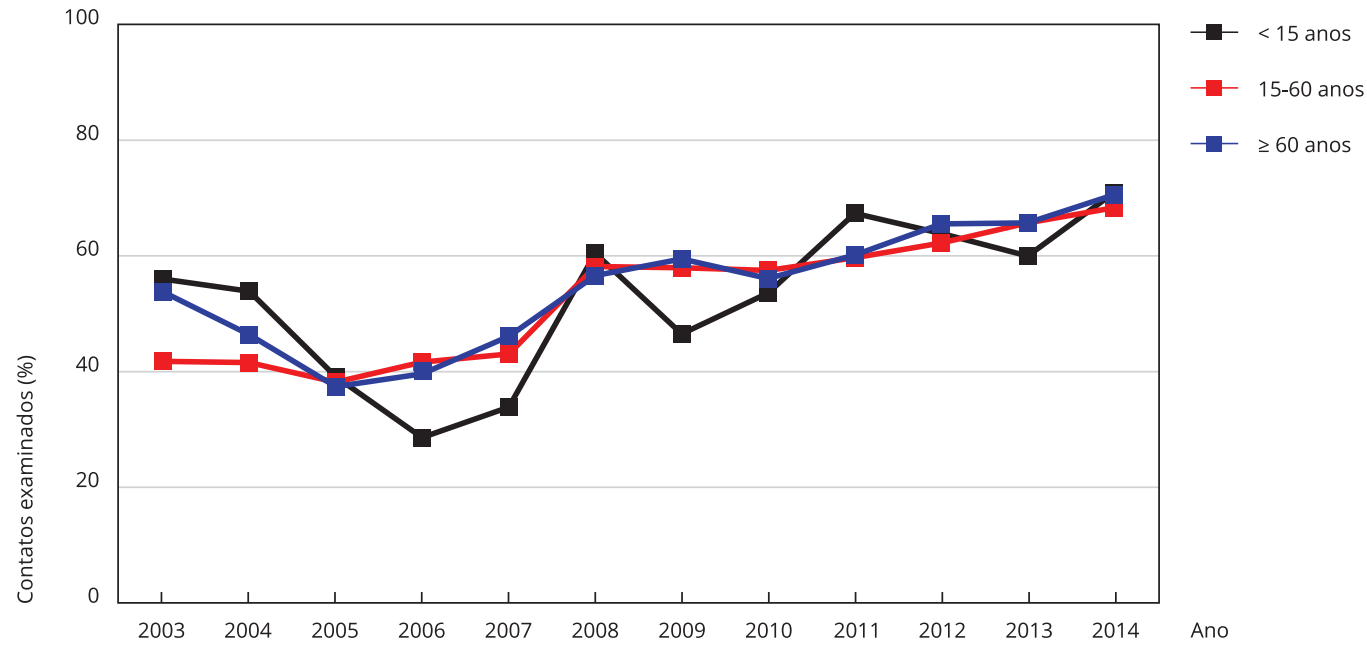

(continua) 
Figura 2 (continuação)

2c) Cor/Raça

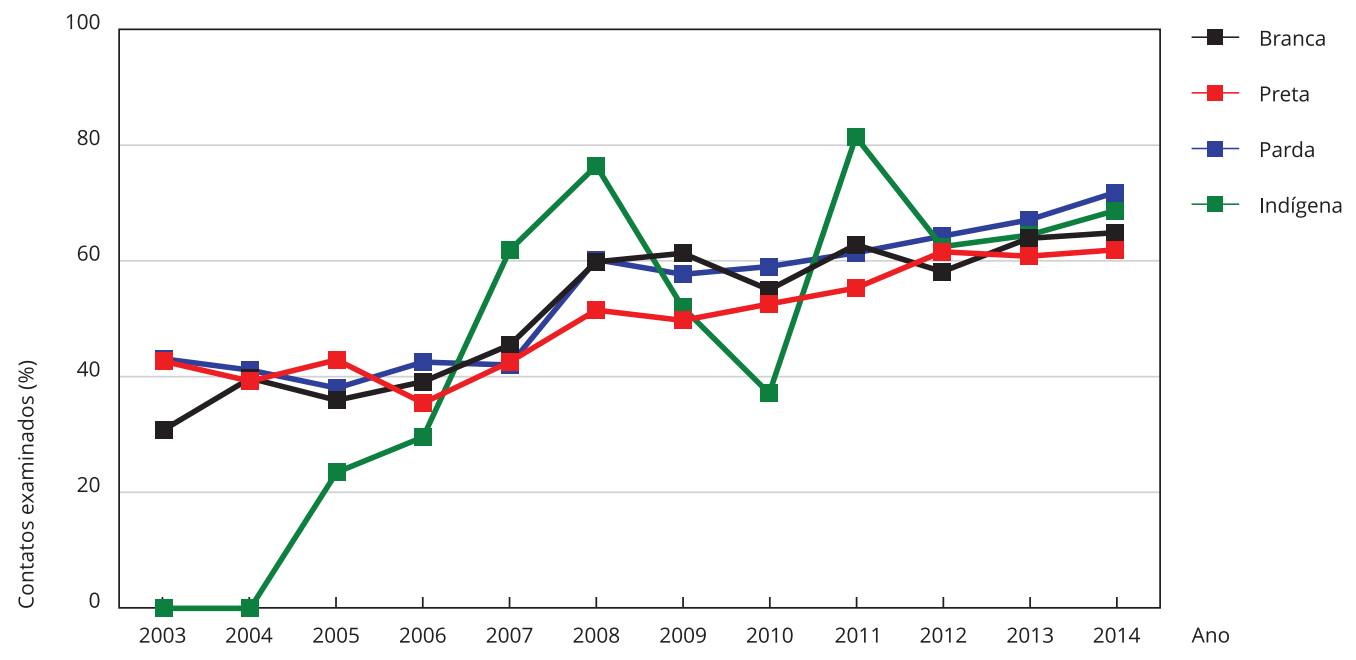

Figura 3

Proporção de contatos examinados entre os registrados, segundo local de residência dos casos-referência de hanseníase. Bahia, Brasil, $2003-2014$.

3a) Residência na capital

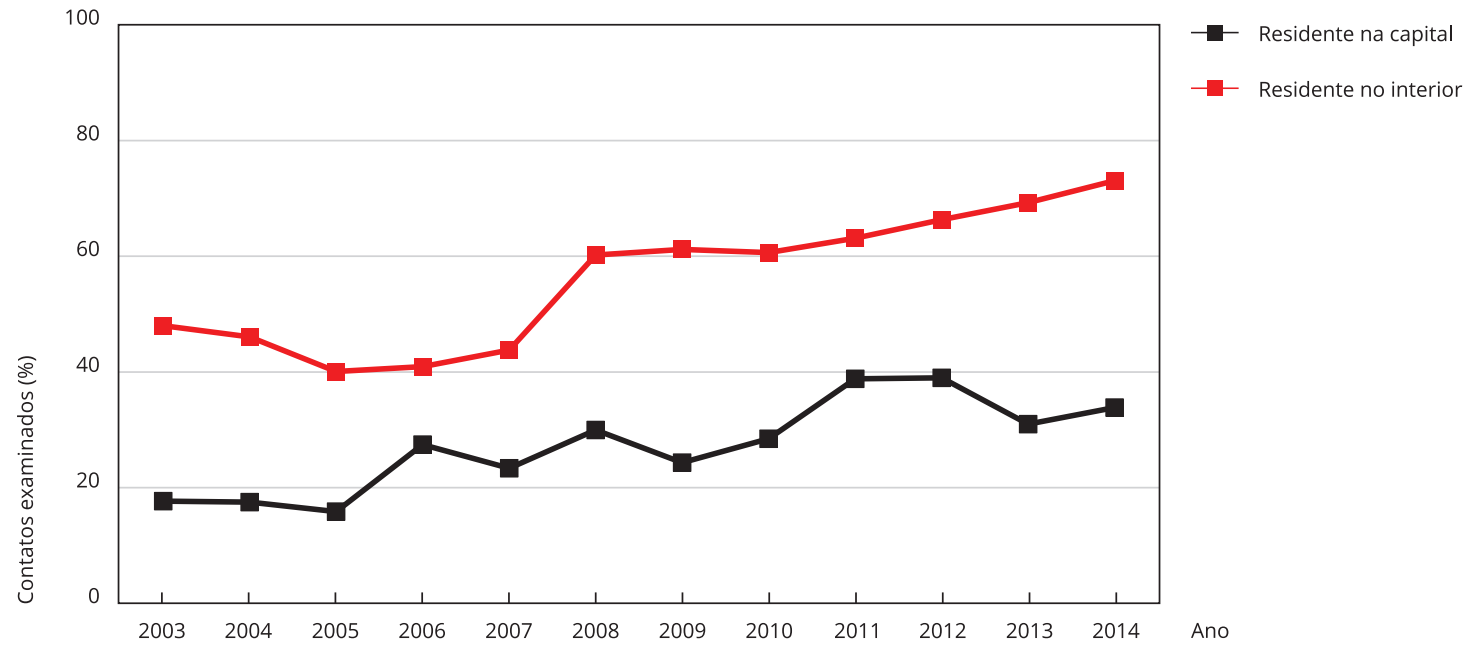

(continua) 
3b) Zona de residência

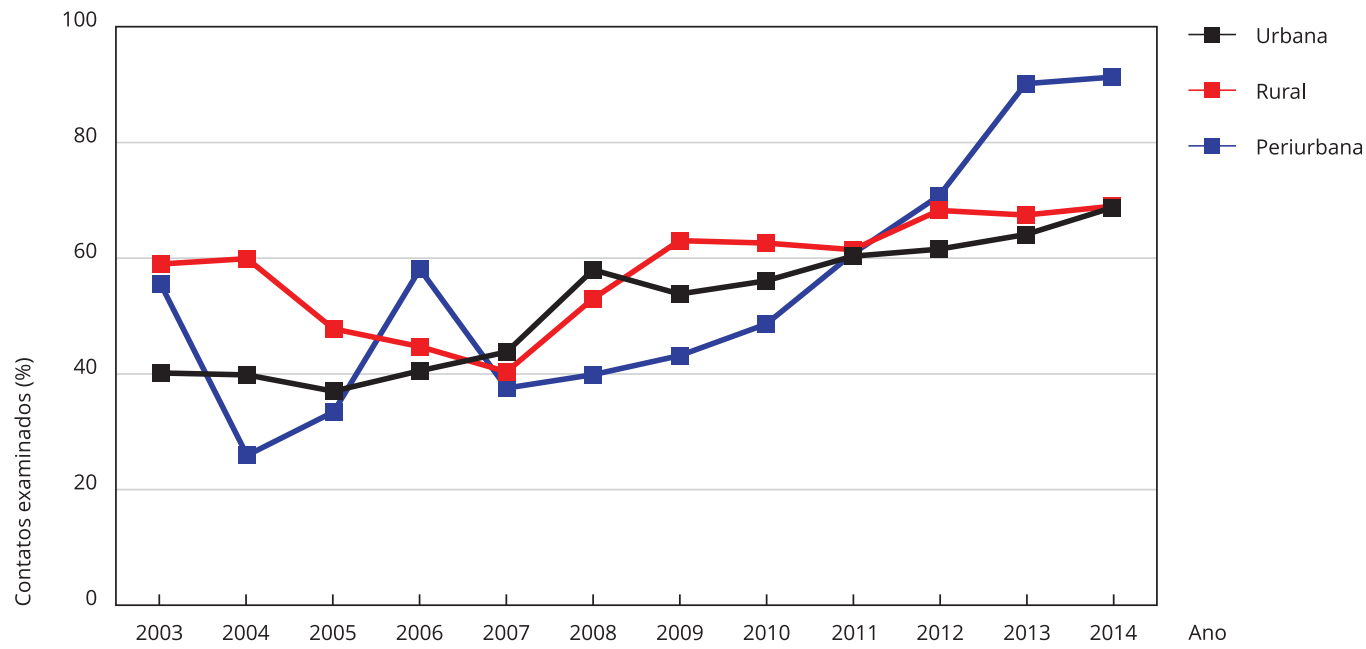

3c) Porte do município

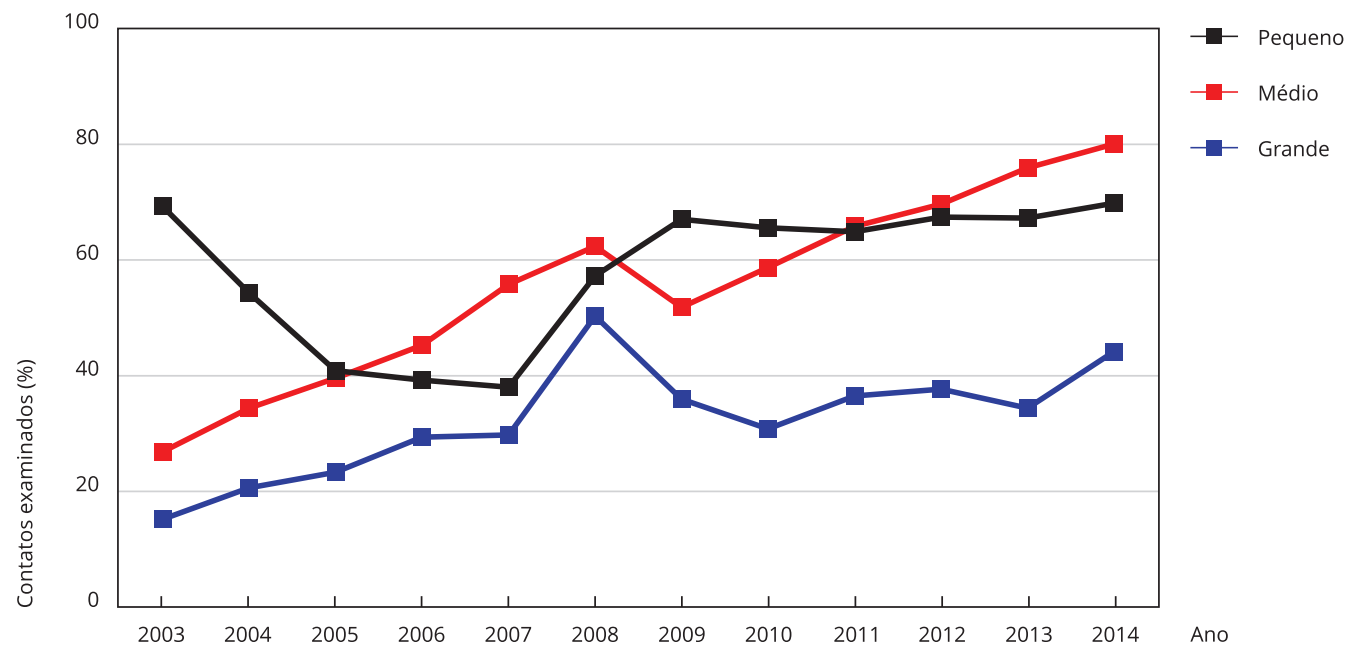

Verificou-se tendência de crescimento menos expressiva entre contatos de CR de raça/cor preta $(\mathrm{APC}=4,7 ; \mathrm{IC} 95 \%: 3,3 ; 6,3)$ quando comparada às demais categorias, com destaque para o aumento expressivo na raça/cor branca (APC = 6,4; IC95\%: 4,3; 8,6) (Tabela 1). Para a raça/cor indígena, a não existência de contatos registrados em alguns anos impossibilitou a análise da tendência por Joinpoint.

Observou-se tendência mais expressiva de crescimento na proporção de contatos examinados em $\mathrm{CR}$ que morava na capital do estado (APC $=7,3$; IC95\%: 3,3; 11,4) quando comparada ao do interior $(\mathrm{APC}=5,6$; IC95\%: 3,$8 ; 7,5)$. Tendência de crescimento menos expressiva foi observada quando o CR morava na zona rural, com duas tendências definidas: 2003-2006 (APC = -8,9; IC95\%: -23,5; 8,4) sem tendência definida e 2006-2014 (APC = 6,1; IC95\%: 2,2; 10,0) com tendência de aumento. CR residentes em área periurbana apresentaram tendência de crescimento expressivo (APC = 8,0; IC95\%: $3,2 ; 13,1)$ (Tabela 1). 


\section{Tabela 1}

Variação Percentual Anual (APC) ou Variação Percentual Anual Média (AAPC) da proporção de contatos examinados relacionados aos registrados, segundo variáveis sociodemográficas dos casos-referência de hanseníase. Bahia, Brasil, 2003-2014.

\begin{tabular}{|c|c|c|c|c|c|}
\hline \multirow[t]{2}{*}{ Indicadores } & \multicolumn{3}{|c|}{ Tendências } & \multicolumn{2}{|c|}{ Período total } \\
\hline & Período & APC & IC95\% & AAPC & IC95\% \\
\hline \multicolumn{6}{|l|}{ Sexo/Gênero } \\
\hline \multirow[t]{3}{*}{ Masculino } & 2003-2005 & $-14,0$ * & $-24,3 ;-2,4$ & 4,9 * & 3,$0 ; 6,8$ \\
\hline & $2005-2008$ & 14,2 * & 0,$2 ; 30,1$ & & \\
\hline & $2008-2014$ & 4,0 * & 2,$1 ; 5,9$ & & \\
\hline Feminino & $2003-2014$ & 5,3 * & 3,$4 ; 7,3$ & 5,3 * & 3,$4 ; 7,3$ \\
\hline \multicolumn{6}{|c|}{ Faixa etária (anos) } \\
\hline$<15$ & $2003-2014$ & 3,8 & $-0,1 ; 7,8$ & 3,8 & $-0,1 ; 7,8$ \\
\hline $15-60$ & 2003-2014 & 5,4 * & 3,$9 ; 6,9$ & 5,4 * & 3,$9 ; 6,9$ \\
\hline \multirow[t]{3}{*}{$>60$} & 2003-2005 & $-18,2 *$ & $-31,1 ;-3,0$ & $5,0 *$ & 2,$7 ; 7,3$ \\
\hline & $2005-2008$ & 7,1 * & 4,$3 ; 9,9$ & & \\
\hline & 2008-2014 & 4,1 * & 1,$6 ; 6,7$ & & \\
\hline \multicolumn{6}{|l|}{ Raça/Cor } \\
\hline Branca & 2003-2014 & 6,4 * & 4,$3 ; 8,6$ & 6,4 * & 4,$3 ; 8,6$ \\
\hline Preta & 2003-2014 & 4,7 * & 3,$3 ; 6,3$ & 4,7 * & 3,$3 ; 6,3$ \\
\hline Parda & 2003-2014 & 5,8 * & 4,$1 ; 7,5$ & 5,8 * & 4,$1 ; 7,5$ \\
\hline Indígena & - & - & - & - & - \\
\hline \multicolumn{6}{|c|}{ Residência na capital } \\
\hline Sim & 2003-2006 & 7,3 * & 3,$3 ; 11,4$ & 7,3 * & 3,$3 ; 11,4$ \\
\hline Não & 2003-2005 & 5,6 * & 3,$8 ; 7,5$ & $5,6 *$ & 3,$8 ; 7,5$ \\
\hline \multicolumn{6}{|c|}{ Zona de reisdência } \\
\hline Urbana & 2003-2014 & 5,8 * & 4,$3 ; 7,3$ & 5,8 * & 4,$3 ; 7,3$ \\
\hline \multirow[t]{2}{*}{ Rural } & 2003-2006 & $-8,9$ & $-23,5 ; 8,4$ & 2,8 * & 0,$3 ; 5,3$ \\
\hline & $2006-2014$ & 6,1 * & 2,$2 ; 10,0$ & & \\
\hline Periurbana & 2003-2014 & 8,0 * & 3,$2 ; 13,1$ & 8,0 * & 3,$2 ; 13,1$ \\
\hline \multicolumn{6}{|c|}{ Porte do município } \\
\hline \multirow[t]{3}{*}{ Pequeno } & 2003-2005 & $-21,6$ & $-50,2 ; 23,5$ & 2,8 & $-0,8 ; 6,4$ \\
\hline & $2005-2009$ & 6,7 * & 2,$1 ; 11,5$ & & \\
\hline & 2009-2014 & 0,7 & $-0,5 ; 2,0$ & & \\
\hline \multirow[t]{2}{*}{ Médio } & $2003-2007$ & 17,6 * & 6,$6 ; 29,8$ & 8,4 * & 6,$1 ; 10,7$ \\
\hline & 2007-2014 & $5,5 *$ & 2,$4 ; 8,7$ & & \\
\hline \multirow[t]{2}{*}{ Grande } & 2003-2008 & 20,2 * & 10,$1 ; 31,1$ & $6,1 *$ & 1,$2 ; 11,2$ \\
\hline & $2008-2014$ & $-1,2$ & $-6,0 ; 3,9$ & & \\
\hline
\end{tabular}

IC95\%: intervalo de 95\% de confiança.

* Significativamente diferente de $0(p<0,05)$.

Duas tendências de aumento da proporção de exame de contatos para CR residentes em cidades de médio porte: a primeira de 2003-2007 (APC = 17,6; IC95\%: 6,6; 29,8) e a segunda de 2007-2014 (APC = 5,5; IC95\%: 2,4; 8,7). Para CR que residiam em cidades de grande porte verificou-se tendência de crescimento de 2003-2008 (APC = 20,2; IC95\%: 10,1; 31,1), seguida de tendência não definida entre 2008-2014 (APC =-1,2; IC95\%: -6,0; 3,9). Já para as cidades de pequeno porte foram identificadas três tendências, a primeira de 2003-2005 (APC = -21,6; IC95\%: -50,2; 23,5) sem tendência definida, a segunda de 2005-2009 (APC = 6,7; IC95\%: 2,1; 11,5) com tendência de crescimento e a última de 2009-2014 (APC = 0,7; IC95\%: -0,5; 2,0) também sem tendência definida (Tabela 1).

$\mathrm{Na}$ análise espacial, a maioria dos municípios de todas as regiões do estado apresentava proporção de contatos examinados entre registrados $\leq 75 \%$ no período de 2003-2008. De 2009-2014, houve redução no número de municípios com desempenho precário, com destaque para as regiões 
extremo-sul e nordeste. O mapa referente ao período completo (2003-2014) confirma o padrão de precariedade desse indicador, inclusive com municípios que não tiveram nenhum contato examinado ao longo da série histórica (Figura 4).

A avaliação de autocorrelação espacial por Moran local evidenciou no primeiro período a existência de pequenos clusters nas regiões leste, nordeste e sul do estado, com municípios com elevada proporção de contatos não avaliados. Em contraponto, municípios localizados nas regiões norte, centro-norte, sul e sudoeste do estado apresentaram clusters com baixa proporção de contatos não examinados. No segundo período, houve aumento do número de municípios do cluster na região nordeste, com baixo desempenho do indicador, além de ampliação do cluster com bom desempenho nas regiões centro-norte e sudoeste. Esse padrão é verificado no mapa referente ao período total da coorte analisada (Figura 4).

\section{Discussão}

O estudo revela a precariedade no desempenho das ações de vigilância de contatos de casos de hanseníase no Estado da Bahia, em particular quando o CR residia em Salvador, regiões periurbanas e em cidades de grande porte. Embora a tendência temporal da proporção de contatos examinados entre os registrados tenha sido de incremento, ainda está distante do ideal. É importante ressaltar a existência de dinâmicas diferentes para a tendência desse indicador, com aumento menos expressivo da proporção de contatos examinados quando o CR é do sexo masculino, de raça/cor preta, residente na zona rural e em cidades de pequeno porte. A análise espacial revelou que em todas as regiões do estado a maioria dos municípios apresentou desempenho precário dessa ação.

A proporção de contatos examinados de CR de hanseníase mede a capacidade dos serviços de saúde de realizar vigilância. Tendo em vista a importância epidemiológica dos contatos em hanseníase e da detecção oportuna de casos novos para controle 2, os baixos valores quando o CR residia na capital e em áreas periurbanas das cidades baianas de grande porte podem indicar limitação de acesso a serviços e ações de saúde. Ainda que os quatro maiores municípios baianos (Salvador, Feira de Santana, Vitória da Conquista e Itabuna) não façam parte dos principais clusters de detecção de hanseníase deste estado 20, esta situação dificulta o alcance do controle, contribuindo para endemia oculta e diagnóstico tardio 1,2,21.

Um estudo conduzido em Salvador verificou que a distribuição intraurbana dos casos de hanseníase em crianças com menos de 15 anos de idade, revelou que a transmissão de Mycobacterium leprae estava estabelecida no território desta cidade de modo ativo e intenso, principalmente em bairros periféricos, a exemplo daqueles localizados nos Distritos Sanitários do Subúrbio Ferroviário, São Caetano/Valéria e Itapuã 22. Em outro estudo na capital foi possível reconhecer o longo itinerário terapêutico até a realização do diagnóstico da hanseníase, principalmente pela falta de capacitação de médicos de unidades de saúde, mas também por desconhecimento da população, estigma e preconceito existentes 23 . Os resultados desses estudos corroboram com os achados do presente trabalho, reforçando possíveis falhas no desenvolvimento de medidas de vigilância e controle na capital do Estado da Bahia, distanciando do real controle da hanseníase 21,24.

$\mathrm{O}$ fato de a tendência de crescimento da proporção de contatos examinados ser menos expressiva quando o CR é do sexo masculino, demonstra a importância das análises que envolvem a temática de gênero. Essa questão é amplamente reconhecida em termos da influência em processos de autocuidado e adoecimento 24,25. Outros trabalhos reforçam a importância dessa abordagem, em particular ao evidenciarem maior detecção de casos novos entre homens, assim como a ocorrência expressiva de diagnóstico tardio nesta população, inclusive no Estado da Bahia 18,20,24.

A nota informativa do Ministério da Saúde de 2016 alertou para a importância de exame qualificado e sistemático para a triagem da hanseníase entre homens e idosos 26. Diante desse cenário e considerando-se as recomendações da OMS, a análise epidemiológica por sexo/gênero torna-se essencial para fomentar o planejamento de políticas públicas para o enfretamento da hanseníase. O "ser" homem e o "ser" mulher necessitam ser discutidos dentro de uma perspectiva de redução das diferenças de acesso e atenção à saúde por possível desigualdade de gênero ${ }^{27}$. 


\section{Figura 4}

Distribuição espacial da proporção de contatos não examinados entre registrados por município e autocorrelação espacial por Moran Local. Bahia, Brasil, nos períodos de 2003-2008, 2009-2014 e 2003-2014.

4a) $2003-2008$

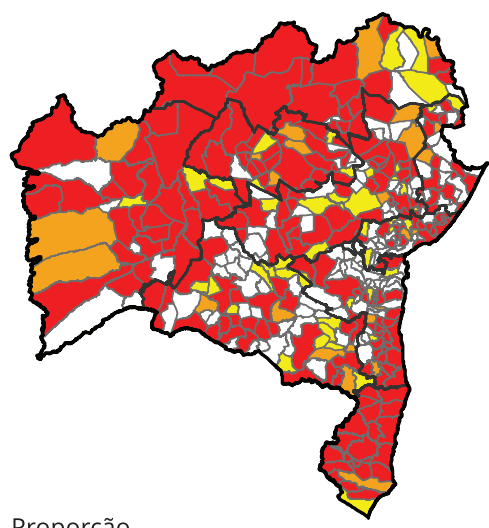

Proporção

$\square$ Ausente

$\square 90,0$

$\square 75,1-89,9$

75,0 4b) $2009-2014$

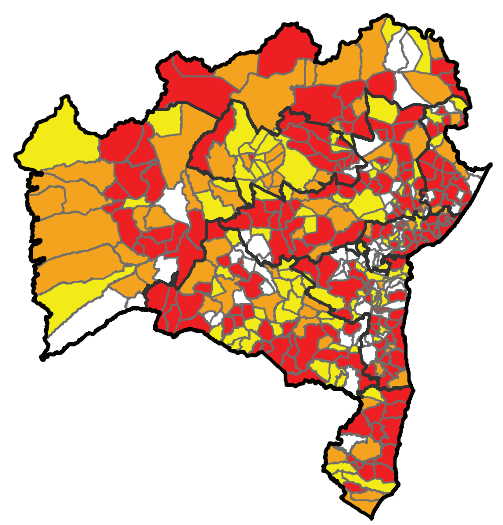

4e) 2009-2014

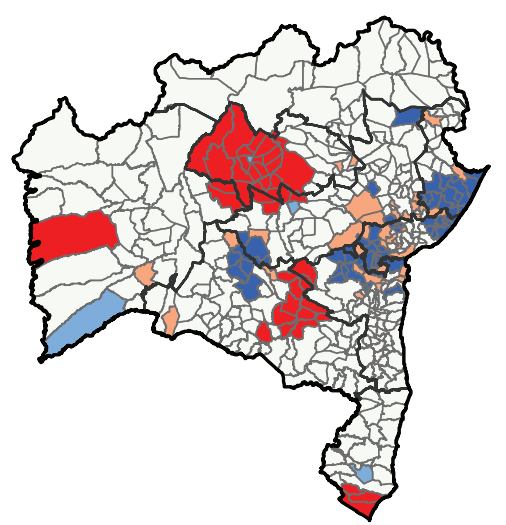

4c) $2003-2014$

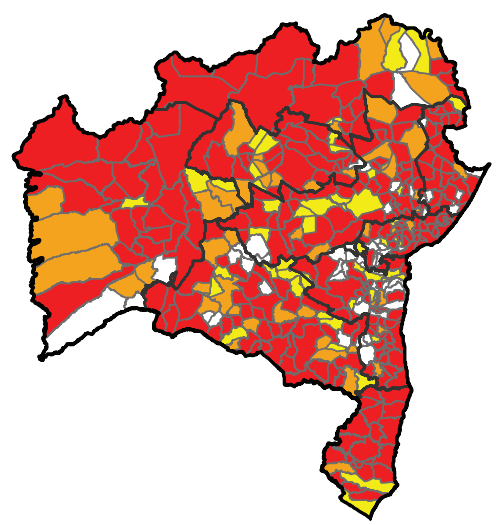

4f) $2003-2014$

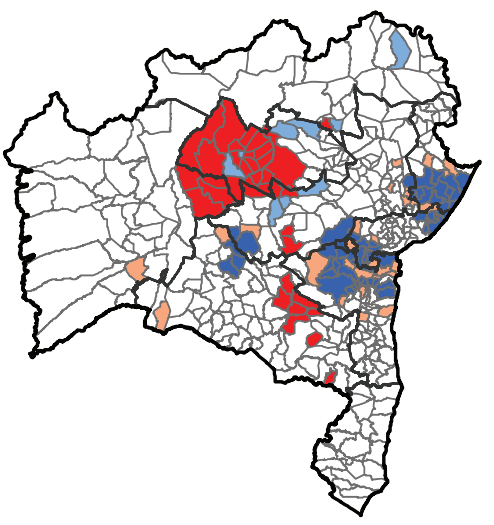

Índice Moran Local

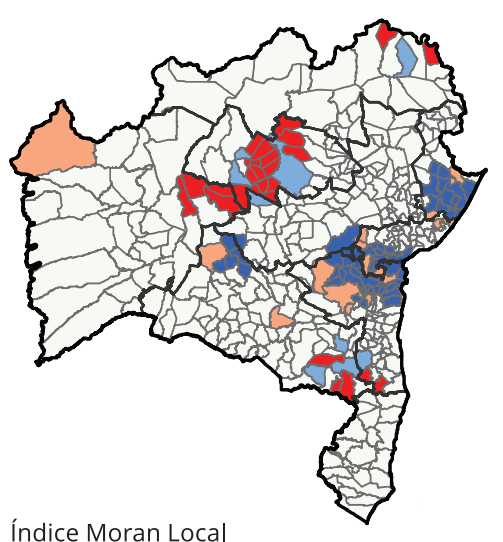

$\square$ Não significante

$\square$ Alto-Alto

$\square$ Alto-Baixo

$\square$ Baixo-Alto

Baixo-Baixo

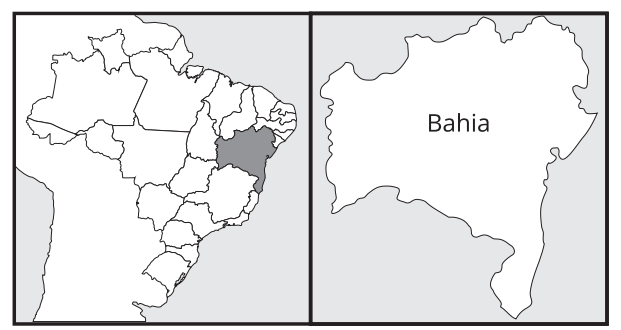


Quando o CR é uma criança menor de 15 anos de idade, a tendência temporal de incremento da proporção de contatos examinados que ocorreu para todas as demais faixas etárias não foi detectada, constatando ausência de tendência em toda a série histórica. Como a existência no espaço domiciliar e comunitário de casos em crianças remete à circulação ativa de $M$. leprae 1,2,28, estas situações deveriam ser priorizadas na vigilância do contato.

Outro estudo revelou tendência temporal de redução de casos de hanseníase na população geral no Estado da Bahia, o que não ocorreu entre menores de 15 anos 20. Dessa forma, a baixa cobertura de avaliação de seus contatos estabelece condição favorável à sustentação da dinâmica de transmissão da doença podendo, inclusive, manter a ocorrência de casos novos em outras gerações da mesma família 29. Um estudo realizado em Salvador revelou que 52,6\% dos casos em menores de 15 anos reconheceram contatos que adoeceram no espaço intradomicílio e 25\% no ambiente de convívio social 22.

O maior risco de transmissão torna esses contextos prioritários para o desenvolvimento das ações de vigilância e controle 1,2. Dessa forma, o seguimento longitudinal de famílias com ocorrência de casos de hanseníase, principalmente mediante o diagnóstico em crianças, é estratégico para a interrupção da transmissão e para o monitoramento da qualidade das ações de vigilância 22,28.

O fato de CR de raça/cor preta apresentarem proporção de contatos examinados com crescimento menos expressivo sinaliza possível situação de desigualdade. Remete-se à expressão histórica no Brasil de um contexto que mantém essas populações em situação de maior vulnerabilidade social, individual e programática 27 . Em conjunto com outros determinantes sociais, reforça o caráter de doença negligenciada da hanseníase, com forte vinculação à pobreza 7,12,30.

No cenário brasileiro, o Boletim Epidemiológico do Ministério da Saúde direcionado para avaliar indicadores de saúde com base na variável raça/cor reafirma as desigualdades persistentes. Baseandose nesse documento, dos 31.064 casos notificados em 2014, 21.554 ocorreram na população de raça/ cor preta e parda. Entre os casos com grau 2 de incapacidade física, 70\% (1.358) foram em pessoas de raça/cor preta. A Região Nordeste notificou o maior número de casos com grau 2 de incapacidade, indicando diagnóstico tardio 31. Desse modo, considerando a posição social de negros no contexto baiano, a intervenção sobre mecanismos de estratificação social torna-se crucial para o enfrentamento desta condição de iniquidades em saúde 27,30 .

A verificação de que CR residente em cidades de pequeno porte ou de zona rural do Estado da Bahia, a tendência de aumento é menos expressiva na proporção de contatos examinados. Diferentes barreiras que dificultam o acesso à atenção e ao cuidado em saúde podem estar presentes. Estudos que integrem a avaliação do acesso são estratégicos, tanto para a pessoa com a doença quanto para seus contatos 32,33 .

Ter acesso remete a dimensões específicas que descrevem a adequação entre usuários e o sistema de atenção, possibilitando consumir tecnologia de saúde necessária para melhorar e prolongar a vida 34 . É importante destacar que para os contatos de hanseníase o acesso durante o seu atendimento deve incluir processos de aconselhamento, anamnese dirigida a sinais e sintomas da doença, educação em saúde, exame dermatoneurológico, vacinação com BCG e promoção de autonomia 2,5.

Além da análise de tendência temporal, a distribuição espacial do indicador em análise revelou que as regiões norte e extremo-sul da Bahia destacam-se pelo elevado número de municípios com baixo desempenho. Da mesma forma, um trabalho recente com análise da taxa de detecção de casos novos de hanseníase demonstrou que nessas regiões encontra-se o maior número de municípios hiperendêmicos, inclusive para a ocorrência de casos em menores de 15 anos de idade e com grau 2 de incapacidade física 21. Estudos de cluster no Brasil também reafirmaram que essas áreas estão associadas ao maior risco de ocorrência de casos 35,36. Portanto, torna-se essencial a intensificação das ações de vigilância de contatos nessas áreas historicamente endêmicas no estado para fortalecer o controle.

Por outro lado, quando observada a autocorrelação espacial, a região nordeste do Estado da Bahia apresentou o maior agregado de municípios relacionados ao baixo desempenho deste indicador. Destaca-se que essa região reúne o maior número de municípios com baixa endemicidade 21. Assim, a baixa detecção de casos novos de hanseníase e a precariedade da proporção de contatos examinados nessa região revelam a importância de estudos adicionais para avaliar o acesso e a qualidade das ações de vigilância e controle dessas populações 1,2,5,21. O fato de terem sido verificados municípios onde não foram registrados exames de contatos também reafirma essa necessidade. 
As discussões até então são essenciais para compreender e planejar ações efetivas para a mudança do cenário apresentado, embora persistam importantes lacunas sobre fatores associados às dificuldades para a realização das ações de vigilância de contatos 37,38,39. No entanto, outras questões também necessitam ser discutidas, como a operacionalização da ampliação das ações de vigilância para outras populações-alvo, ainda que não residam no mesmo domicílio 14,24. No Brasil, em 2016, as diretrizes de vigilância da hanseníase ampliaram o conceito incorporando a dimensão de contato social, sem orientar claramente a operacionalização deste conceito e de como compor indicadores que insiram esta população 2 . Foram incluídos vizinhos, colegas de trabalho e de escola, entre outros, que deveriam ser investigados de acordo com o grau e tipo de convivência 2.

Dessa forma, embora a inclusão do contato social seja um avanço, torna-se fundamental discutir como operacionalizar essas ações no contexto local, de uma forma técnica e ética, em especial quando são considerados registro, sigilo e privacidade. Protocolos éticos para a abordagem necessitam ser pensados, considerando inclusive o estigma associado e os complexos desafios para o controle 40 .

Outra alteração das novas diretrizes faz referência à orientação de que todos os contatos que não estão doentes sejam avaliados anualmente durante cinco anos 2. Considerando o longo período de incubação, torna-se essencial o seguimento dos contatos, reforçando a necessidade de fortalecer o processo de educação em saúde em que a pessoa acometida assume protagonismo sobre o seu cuidado.

A detecção de novos casos de hanseníase deve ser potencializada para além do exame de contatos. Ações mais ativas de vigilância, incluindo exames de coletividade, inquéritos e campanhas, também devem ser mantidas. Da mesma forma, a deteç̧ão passiva também necessita ser prioridade, garantindo atenção qualificada às demandas que chegam às unidades de saúde espontaneamente ou encaminhadas por outros serviços 2 .

O espaço prioritário para o desenvolvimento dessas ações são as unidades de APS 2. No entanto, existem desafios a ser superados para ampliar o acesso e a qualidade desses serviços 15,32, principalmente para as populações de maior vulnerabilidade. O Estado da Bahia, por exemplo, embora tenha apresentado ampliação significativa na cobertura populacional pela Estratégia Saúde da Família, variando de 16,2\% em 2001 para 68,9\% em 2014, persiste ao longo do período estudado com grande número de municípios com padrão insatisfatório de exames de contatos 16,41. Dessa forma, também é urgente pensar em indicadores que traduzam não apenas a cobertura, mas também a qualidade da ação, inclusive do exame dermatoneurológico, que por sua especificidade requer conhecimento e técnicas específicas 2.

Por ser um estudo de base de dados secundários, existem limitações inerentes, como aquelas vinculadas à inconsistência e não completitude. Especificamente nos campos referentes ao número de contatos registrados e avaliados, é relativamente recente o bloqueio para que o número de contatos examinados não extrapolasse o total de registados. Além disso, como os contatos podem chegar aos serviços em diferentes momentos, é possível que a ficha de acompanhamento do SINAN não seja adequadamente atualizada pelos profissionais de saúde. No entanto, o fato de ser uma série histórica de 11 anos de base estadual justifica a sua plena utilização, aliado às diferentes técnicas empregadas. O rigor usado na análise dos dados ressalta aspectos significativos no estudo.

\section{Conclusão}

O Estado da Bahia apresenta tendência de crescimento da proporção de contatos de casos de hanseníase examinados ao longo de 11 anos do estudo. No entanto, persiste com parâmetros de precariedade do ponto de vista operacional, com cobertura aquém do ideal para o controle. Ressalta-se a necessidade de se ampliar a discussão sobre os fatores relacionados ao pior desempenho dos serviços de saúde, em particular na capital do estado, nas regiões periurbanas e em cidades de grande porte.

Padrões de tendência de aumento menos significativo ou de ausência de tendência da proporção de contatos examinados entre examinados quando o CR é do sexo masculino, menor de 15 anos, de raça/cor preta e residente na zona rural, reforçam aspectos de vulnerabilidade social, individual e operacional. Reforçam a necessidade de se desenvolver estratégias específicas para esses grupos populacionais, com vistas à maior efetividade das ações de controle. 
A análise espacial do indicador proporção de contatos examinados entre os registrados traduz um grande número de municípios com desempenho insatisfatório. Além disso, a formação de pequenos clusters de baixo desempenho em áreas de menor endemicidade pode indicar associação entre baixos coeficientes de casos novos com a baixa cobertura do exame de contatos. Desse modo, estratégias para ampliar a cobertura das ações de vigilância do contato devem ser direcionadas para todas as regiões do estado, fato que amplia ainda mais o desafio para um estado brasileiro com 417 municípios.

\section{Colaboradores}

E. A. Souza e A. N. Ramos Jr. contribuíram com a concepção e delineamento do estudo, análise e interpretação dos dados, redação do artigo, revisão crítica relevante do conteúdo intelectual e aprovação final da versão a ser publicada. A. F. Ferreira e J. Heukelbach contribuíram com a análise e interpretação dos dados, redação do artigo, revisão crítica relevante do conteúdo intelectual e aprovação final da versão a ser publicada. M. S. A. P. Pinto, H. X. Oliveira e J. C. Barbosa contribuíram com a análise e interpretação dos dados, revisão crítica relevante do conteúdo intelectual e aprovação final da versão a ser publicada.

\section{Referências}

1. World Health Organization. Global leprosy update, 2017: reducing the disease burden due to leprosy. Wkly Epidemiol Rec 2018; 93:44556.

2. Departamento de Vigilância das Doenças Transmissíveis, Secretaria de Vigilância em Saúde, Ministério da Saúde. Diretrizes para vigilância, atenção e eliminação da hanseníase como problema de saúde pública: manual técnico-operacional. Brasília: Ministério da Saúde; 2016.

3. World Health Organization. Global leprosy strategy 2016-2020: accelerating towards a leprosy-free world. http://apps.searo.who.int/ PDS_DOCS/B5233.pdf (acessado em 14/Jan/ 2019).

4. Freitas LRS, Duarte EC, Garcia LP. Análise da situação epidemiológica da hanseníase em uma área endêmica no Brasil: distribuição espacial dos períodos 2001-2003 e 2010-2012. Rev Bras Epidemiol 2017; 20:702-13.

5. Smith CS, Noordeen SK, Richardus JH, Sansarricq H, Cole ST, Soares RC, et al. A strategy to halt leprosy transmission. Lancet Infect Dis 2014; 14:96-8.

6. Penna ML, Penna GO, Iglesias PC, Natal S, Rodrigues LC. Anti-PGL-1 positivity as a risk marker for the development of leprosy among contacts of leprosy cases: systematic review and meta-analysis. PLoS Negl Trop Dis 2016; 10:e0004703.

\section{Informações adicionais}

ORCID: Eliana Amorim de Souza (0000-00029653-3164); Anderson Fuentes Ferreira (00000002-1816-9459); Maria Solange Araújo Paiva Pinto (0000-0002-3664-3927); Jorg Heukelbach (0000-0002-7845-5510); Héllen Xavier Oliveira (0000-0002-2814-0182); Jaqueline Caracas Barbosa (0000-0001-6401-2244); Alberto Novaes Ramos Jr. (0000-0001-7982-1757).

7. Mieras LF, Anand S, van Brakel WH, Hamilton HC, Martin Kollmann KH, Mackenzie C, et al. Neglected Tropical Diseases, Cross-Cutting Issues Workshop, 4-6 February 2015, Utrecht, the Netherlands: meeting report. Int Health 2016; 8 Suppl 1:i7-11.

8. Hofstraat K, Van Brakel WH. Social stigma towards neglected tropical diseases: a systematic review. Int Health 2016; 8 Suppl 1:i53-70.

9. Chirwa T, Floyd S, Fine P. Estimating the extent of household contact misclassification with index cases of disease in longitudinal studies using a stochastic simulation model. Glob Health Action 2013; 6:19614.

10. Nicchio MV, Araujo S, Martins LC, Pinheiro AV, Pereira DC, Borges A, et al. Spatial and temporal epidemiology of Mycobacterium leprae infection among leprosy patients and household contacts of an endemic region in Southeast Brazil. Acta Trop 2016; 163:38-45.

11. Sales AM, Leon AP, Düppre NC, Hacker MA, Nery JAC, Sarno EN, et al. Leprosy among patient contacts: a multilevel study of risk factors. PLoS Negl Trop Dis 2011; 5:e1013.

12. Smith W, Aerts A. Role of contact tracing and prevention strategies in the interruption of leprosy transmission. Lepr Rev 2014; 85:2-17.

13. Sarno EN, Duppre NC, Sales AM, Hacker MA Nery JA, Matos HJ. Leprosy exposure, infection and disease: a 25-year surveillance study of leprosy patient contacts. Mem Inst Oswaldo Cruz 2012; 107:1054-9. 
14. Romanholo HSB, Souza EA, Ramos AN, Kaiser ACGCB, Silva IO, Brito AL, et al. Surveillance of intradomiciliary contacts of leprosy cases: perspective of the client in a hyperendemic municipality. Rev Bras Enferm 2018; 71:17581.

15. Oliveira SP. Acessibilidade ao exame de contato de hanseníase na Estratégia de Saúde da Família em Cuiabá, Mato Grosso - Brasil [Dissertação de Mestrado]. Salvador: Instituto de Saúde Coletiva, Universidade Federal da Bahia; 2013.

16. Ministério da Saúde. Hanseníase: situação epidemiológica. http://portalms.saude.gov.br/ saude-de-a-z/hanseniase/situacao-epidemio logica (acessado em 14/Jan/2019).

17. Instituto Brasileiro de Geografia e Estatística. Projeções e estimativas da população do Brasil e das Unidades da Federação. https://cidades. ibge.gov.br/brasil/ba/panorama (acessado em 12/Fev/2019).

18. Instituto de Pesquisa Econômica Aplicada. Atlas do desenvolvimento humano no Brasil, 2013. http://www.atlasbrasil.org.br/2013/pt/ perfil_uf/bahia (acessado em 18/Jan/2019).

19. Anselin L. Local indicators of spatial association - LISA. Geogr Anal 1995; 27:93-115.

20. Souza EA, Ferreira AF, Boigny RN, Alencar $\mathrm{CH}$, Heukelbach J, Martins-Melo FR, et al. Leprosy and gender in Brazil: trends in an endemic area of the Northeast region, 20012014. Rev Saúde Pública 2018; 52:20.

21. Souza EA, Ferreira AF, Boigny R, Heukelbach J, Alencar CH, Ramos Jr. AN. Epidemiology and spatiotemporal patterns of leprosy detection in the State of Bahia, Brazilian Northeast Region, 2001-2014. Trop Med Infect Dis 2018; 3:79.

22. Santos SD. Hanseníase em menores de 15 anos em Salvador (Bahia) [Dissertação de Mestrado]. Salvador: Instituto de Saúde Coletiva, Universidade Federal da Bahia; 2013.

23. Martins PV, Iriart JAB. Itinerários terapêuticos de pacientes com diagnóstico de hanseníase em Salvador, Bahia. Physis (Rio J.) 2014; 24:273-89.

24. Souza EA, Nzundu RF, Fuentes A, Alencar CH, Oliveira MLW, Ramos Jr. AN, et al. Vulnerabilidade programática no controle da hanseníase: padrões na perspectiva de gênero no Estado da Bahia, Brasil. Cad Saúde Pública 2018; 34:e00196216.

25. Schraiber LB, Gomes R, Couto MT. Homens e saúde na pauta da Saúde Coletiva. Ciênc Saúde Colet 2005; 10:7-17.

26. Ministério da Saúde. Nota informativa conjunta - alerta para exame sistemático de hanseníase na população masculina e em idosos. http://portalarquivos2.saude.gov.br/images/ pdf/2016/setembro/06/Nota-InformativaConjunta-n---01--SAS-e-SVS--para-publica---o.pdf (acessado em 14/Jan/2019).

27. Batista LE. Masculinidade, raça/cor e saúde. Ciênc Saúde Colet 2005; 10:71-80.
28. Santos SD, Penna GO, Costa MDCN, Natividade MS, Teixeira MG. Leprosy in children and adolescents under 15 years old in an urban centre in Brazil. Mem Inst Oswaldo Cruz 2016; 111:359-64.

29. Durães SMB, Guedes LS, Cunha MD, Cavaliere FAM, Oliveira MLWDR. Estudo de 20 focos familiares de hanseníase no Município de Duque de Caxias, Rio de Janeiro. An Bras Dermatol 2005; 80 Suppl 3:S295-300.

30. Carvalho AI, Buss PM, Lobato LVC, Noronha JC. Determinantes sociais na saúde, na doença e na intervenção. In: Giovanella L, Escorel S, Lobato LVC, Noronha JC, Carvalho AI, organizadores. Políticas e sistema de saúde no Brasil. 2a Ed. Rio de Janeiro: Editora Fiocruz; 2012. p. 121-42.

31. Secretaria de Vigilância em Saúde, Ministério da Saúde. Indicadores de vigilância em saúde segundo a variável raça/cor, Brasil. Boletim Epidemiológico 2017; 48(4).

32. Arantes CK, Garcia MLR, Filipe MS, Nardi SMT, Paschoal VDA. Avaliação dos serviços de saúde em relação ao diagnóstico precoce da hanseníase. Epidemiol Serv Saúde 2010; 19:155-64.

33. Cunha ABO, Vieira-da-Silva LM. Acessibilidade aos serviços de saúde em um município do Estado da Bahia, Brasil, em gestão plena do sistema. Cad Saúde Pública 2010; 26:725-37.

34. Augusto CS, Souza M. Adesão do comunicante de hanseníase à profilaxia. Saúde Coletiva 2006; 11:85-90.

35. Penna ML, Penna GO. Leprosy frequency in the world, 1999-2010. Mem Inst Oswaldo Cruz 2012; 107 Suppl 1:3-12.

36. Souza C, Rodrigues M. Magnitude, tendência e espacialização da hanseníase em menores de 15 anos no Estado da Bahia, com enfoque em áreas de risco: um estudo ecológico. Hygeia (Uberlândia) 2015; 11:53-65.

37. Sundar RP, Jesudasan K, Mani K, Christian M. Impact of MDT on incidence rates of leprosy among household contacts. Part 1. Baseline data. Int J Lepr Other Mycobact Dis 1989; 57:647-51.

38. Swain J, Mishra S, Jena S. Prevalence of leprosy among household contacts of leprosy cases in western Orissa. Indian J Lepr 2004; 76:1929.

39. van Beers SM, Hatta M, Klatser PR. Patient contact is the major determinant in incident leprosy: implications for future control. Int J Lepr Other Mycobact Dis 1999; 67:119-28.

40. Kumar A, Lambert S, Lockwood DNJ. Picturing health: a new face for leprosy. Lancet 2019; 393:629-38.

41. Ministério da Saúde. Histórico de cobertura da saúde da família. http://dab.saude.gov.br/ portaldab/historico_cobertura_sf.php (acessado em 14/Jan/2019) 


\section{Abstract}

The aim was to analyze the space-time trend in the proportion of contacts examined among those recorded, according to demographic profile of new leprosy cases in the State of Bahia, Brazil, in the 2003-2014 cohort. This was a state populationbased ecological study with temporal and spatial analyses of the proportion of contacts examined among those recorded, according to characteristics of the reference leprosy case, based on the Information System for Notifiable Diseases of the Brazilian Ministry of Health. The time trend analysis was based on Poisson regression (Joinpoints). Spatial analysis used spatial autocorrelation by local indicators of spatial association. Over the course of the historical series, $52.9 \%(55,116 / 104,142)$ of the recorded contacts were examined, with lower proportions in persons $<60$ years of age, indigenous individuals, and residents of large cities (especially the state capital Salvador). There was an upward trend in the proportion of contacts examined, less evident when the reference leprosy case was a male, black, living in the rural area, and in small towns or the state capital. Spatial distribution showed that the majority of the municipalities showed precarious performance, with clusters identified in the North and Far South of the state. Bahia shows precarious performance on contact surveillance, particular in conditions of greater social vulnerability. Additional strategies should be implemented in order to overcome the operational obstacles to contact surveillance, considered essential for interrupting leprosy transmission in the state.

Leprosy; Contact Tracing; Public Health Surveillance; Prevention es Control

\section{Resumen}

El objetivo de este trabajo fue analizar la tendencia espacio-temporal de la proporción de contactos examinados entre quienes están registrados, según su perfil demográfico, como casos nuevos de lepra, diagnosticados en el estado de Bahía, Brasil, en la cohorte 2003-2014. Se trata de un estudio ecológico de base poblacional estatal, con análisis temporal y espacial de la proporción de contactos examinados entre los registrados, según las características del caso referencia de lepra, a partir del Sistema de Información sobre Enfermedades de Notificación Obligatoria del Ministerio de Salud. El análisis de tendencia temporal se basó en la regresión de Poisson por puntos de inflexión (Joinpoints). El análisis espacial utilizó la autocorrelación espacial mediante el Índice Local de Asociación Espacial. A lo largo de la serie histórica, un 52,9\% (55.116/104.142) de los contactos registrados fueron examinados, con una menor proporción entre personas $<60$ años, raza/etnia indígena y residentes en municipios de gran tamaño (en particular en la capital). Se verificó la tendencia de aumento de la proporción de contactos examinados, con menor expresión, cuando el caso referencia de lepra era de sexo masculino, de raza/ color negro, residente en zona rural y en ciudades de pequeño tamaño, en particular en la capital del estado. La distribución espacial demostró que la mayoría de los municipios tuvo un desempeño con parámetro precario, con aglomerados identificados en las regiones Norte y Extremo-Sur. El Estado de Bahía presenta un desempeño precario de las acciones de vigilancia de contactos, en particular ante condiciones de mayor vulnerabilidad social. Deben ser implementadas estrategias adicionales, con el fin de superar obstáculos operacionales para esta acción, considerada esencial para la interrupción de la transmisión de la lepra.

Lepra; Trazado de Contacto; Vigilancia en Salud Pública; Prevención es Control
Recebido em 31/Out/2018

Versão final reapresentada em 13/Mar/2019 Aprovado em 29/Mar/2019 